\title{
Neuromagnetic Somatosensory Responses to Natural Moving Tactile Stimulation
}

\author{
Yung-Yang Lin, Matti Kajola
}

\begin{abstract}
Objective: To explore the somatosensory cortical responses to natural moving tactile stimulation in adult subjects using magnetoencephalography. Methods: We measured cortical somatosensory magnetic evoked fields (SEFs) to moving tactile stimuli by a brush over the right thumb once every $1.5 \mathrm{~s}$ in seven subjects. Electric SEFs with various intensity or simulated jitter were used for comparison. Results: Tactile SEFs in primary somatosensory cortex (SI) consisted of two deflections: $\mathrm{N} 24 \mathrm{mT}$ and P55mT. Electric SEFs consisted of N24mE, P30mE, P40mE, and P55mE. The amplitude of $\mathrm{N} 24 \mathrm{mT}$ was only $34 \% \pm 12 \%$ of $\mathrm{N} 24 \mathrm{mE}$, whereas P55mT and P55mE were of about the same size. With increased jitter or decreased intensity, attenuation of electric SEFs was more clearly found in early deflection than late deflection. Conclusions: Natural moving tactile stimulation produced simpler cortical somatosensory waveforms in comparison with electric SEFs, partly related to less sharp intensity and stimulation jitter with moving tactile stimulation. We propose that of all the afferent fibers conveying the early deflection, the low threshold components participate the generation of the late deflection.
\end{abstract}

RÉSUMÉ: Potentiels somesthésiques neuromagnétiques à la stimulation tactile mobile naturelle. Objectif: Explorer les potentiels corticaux somesthésiques à la stimulation tactile mobile naturelle chez des adultes au moyen de la magnétoencéphalographie. Méthodes: Nous avons mesuré les champs évoqués magnétiques somesthésiques corticaux (SEFs) à la stimulation tactile mobile du pouce droit à toutes les 1.5 secondes chez sept sujets. Les SEFs électriques d'intensité variable ou les secousses simulées ont été utilisés pour fins de comparaison. Résultats: Les SEFs tactiles dans le cortex somesthésique primaire comportaient deux déflexions: N24mT et P55mT. Les SEFs électriques étaient N24mE, P30mE, P40mE et P55mE. L'amplitude de N24mT était de seulement 34\% $\pm 12 \%$ de celle de $\mathrm{N} 24 \mathrm{mE}$, alors que P55mT et P55mE étaient à peu près de la même dimension. Avec l'augmentation des secousses ou la diminution de l'intensité, l'atténuation des SEFs électriques était plus évidente dans la déflexion précoce que dans la déflexion tardive. Conclusions: La stimulation tactile mobile naturelle a produit des ondes somesthésiques corticales plus simples que les SEFs électriques, en partie parce que la stimulation tactile mobile produit une stimulation moins aiguë. Nous proposons que, de toutes les fibres afférentes transmettant la déflexion précoce, les composantes à seuil bas participent à la génération de la déflexion tardive.

Can. J. Neurol. Sci. 2003; 30: 31-35

Magnetoencephalographic recordings allow non-invasive assessment of somatosensory cortical processing. ${ }^{1}$ Electric pulses are commonly applied on peripheral nerves to elicit somatosensory evoked magnetic fields (SEFs). Although such stimuli produce synchronized afferent volleys and clear cortical responses, they are unnatural and activate simultaneously fibers with different conduction velocities, from both deep and superficial receptors. Therefore, several types of more natural stimuli have been introduced: vibration, ${ }^{2-4}$ tapping, ${ }^{5}$ and airpuffs. ${ }^{6-10}$ Vibration and tapping can result in additional stimulation of muscle and joint receptors. The aims of the present study were to explore the cortical responses to natural moving tactile stimuli and compare these responses with electric SEFs.

\section{METHODS}

Somatosensory magnetic evoked fields were recorded from seven healthy right-handed subjects (four males, three females; mean age 30 years, range $26-35$ years). During the recording,

\footnotetext{
From the Integrated Brain Research Unit, Department of Medical Research and Education, and Neurology, Neurological Institute, Taipei Veterans General Hospital; National Yang-Ming University School of Medicine, Taipei, Taiwan (YYL); Brain Research Unit, Low Temperature Laboratory, Helsinki University of Technology, Espoo, Finland (YYL, MK)

ReCeIVEd May 24, 2002. AcCePTED IN Final Form August 12, 2002. Reprint requests to: Yung-Yang Lin, Neurology, Neurological Institute, Taipei Veterans General Hospital, No. 201, Sec 2, Shih-Pai Rd, Taipei 112, Taiwan
} 
the subject was sitting comfortably in a magnetically shielded room with the head leaning against the helmet-shaped neuromagnetometer. All subjects were instructed to keep their eyes fixed forward and to ignore the stimuli.

The palmar skin of the distal phalanx of the right thumb was stimulated with tactile and electric stimuli in successive sequences; the order of conditions was randomized across subjects. We modified a previously described method to stimulate the subject with the experimenter's thumb movements, triggered by electric stimulation of the median nerve of the experimenter, not of the subject. ${ }^{11}$ Figure 1(a) shows the experimental setup for moving tactile stimulation. The subject comfortably kept his or her right hand on a table with the palm up. The experimenter's (Y.Y. Lin) right hand was also on the table, and a brush (diameter $6 \mathrm{~mm}$ ) was fixed on the thumb. The experimenter's median nerve was stimulated at the wrist once every $1.5 \mathrm{~s}$ with $0.3-\mathrm{ms}$ constant current pulses $(9 \mathrm{~mA})$ to produce clear movement of the thumb. The motion of the brush was horizontal to the pad of the subject's thumb. Consequently, the palmar skin of the distal phalanx of the subject's right thumb was stimulated by the brush once every $1.5 \mathrm{~s}$. Care was taken to avoid any change in the contact of the brush and the subject's skin.

We measured optically (NKK optical detector, NIKKAI, OD1BRN) the movement pattern of the brush and found out that the brush moved upward for $15 \pm 0.6 \mathrm{~mm}$ (mean \pm standard error of the mean $(\mathrm{SEM}))$ at a velocity of $88 \pm 4.6 \mathrm{~cm} / \mathrm{s}$, and then returned to the stationary position $250-300 \mathrm{~ms}$ later. The time lag beween the median nerve stimulus and the initial movement of the brush was $25.1 \pm 3.9 \mathrm{~ms}$; this delay was subtracted from the latencies of the tactile SEFs.

For electric SEFs, 0.3-ms constant current pulses were delivered once every $1.5 \mathrm{~s}$ with bipolar electrodes (pad separation $25 \mathrm{~mm}$ ) on the palmar skin of the distal phalanx of the subject's right thumb. The stimulus intensity was 2 times sensory threshold $(\mathrm{ST})$. Sensory threshold $(3.3 \pm 0.2 \mathrm{~mA}$; range $2.8-4.0$ $\mathrm{mA}$ ) was determined by the lowest intensity with consistent tactile perception around the stimulated area. In Subject 1, SEFs were also recorded with intensities at both $1 \mathrm{ST}$ and $3 \mathrm{ST}$.

The SEFs were recorded with a helmet-shaped 306-channel detector array (Vectorview ${ }^{\mathrm{TM}}$, Neuromag Ltd, Helsinki, Finland) which comprises 102 identical triple sensor elements. ${ }^{12}$ Each sensor element consists of two orthogonal planar gradiometers and one magnetometer coupled to multi-SQUIDs (Superconducting Quantum Interference Devices) and thus provides three independent measures of the magnetic field. The exact location of the head with respect to the sensors was found by measuring magnetic signals produced by currents led to four head indicator coils placed at known sites on the scalp. The locations of the coils with respect to anatomical landmarks on the head were determined with a three-dimensional (3-D) digitizer to allow alignment of the magnetoencephalographic and magnetic resonance image coordinate systems. Magnetic resonance images of the subjects' brains were acquired with a $1.5-\mathrm{T}$ Siemens Magnetom ${ }^{\mathrm{TM}}$ scanner.

The signals were bandpass filtered $(0.03-200 \mathrm{~Hz})$ and digitized at $600 \mathrm{~Hz}$. The analysis period of $800 \mathrm{~ms}$ included a prestimulus baseline of $200 \mathrm{~ms}$, and about 200 responses were averaged for each condition. Epochs coinciding with signals exceeding $150 \mu \mathrm{V}$ in the simultaneously recorded vertical electro-oculogram were automatically rejected from the analysis.

The source analysis was based on signals recorded by the 204 gradiometers. To identify the sources of the measured signals, we visually searched those channels with signal deflections clearly exceeding the prestimulus background level and selected the time windows of cortical responses for further analysis. During these time windows (from the beginning of the deflection to its return to the baseline level) the magnetic field patterns were first visually surveyed in $2 \mathrm{~ms}$ steps to create the initial guess of the number of active sources within that time period and to estimate the stability of the dipolar magnetic field pattern. The equivalent current dipoles (ECDs), best describing the measured data, were found by a least-squares search using subsets of 16-18 channels around the maximum responses. These calculations resulted in the 3-D locations, orientations, and strengths of the ECDs in a spherical conductor model, which was based on subject's magnetic resonance images. Goodness-of-fit of the model was calculated and only ECDs explaining more than $85 \%$ of the field variance at selected periods of time over a subset of channels were used for further analysis. ${ }^{12,13}$

In order to evaluate how a possible jitter in stimulus timing would change the waveforms of averaged SEFs, we made a simple simulation. We recorded 200 epochs of somatosensory responses to strong electric stimulation $(9 \mathrm{~mA}$; interstimulus interval $1.5 \mathrm{~s}$ ) of the right median nerve at the wrist of one author (Y.Y. Lin). The individual epochs were then translated randomly in time with constant probability density distribution between either -5 to $+5,-7.5$ to +7.5 , or -12.5 to $+12.5 \mathrm{~ms}$. The individual epochs were then averaged together. The best dipoles for explaining these averaged responses were obtained for comparison of source waveforms between different jitter distributions.

Statistical significance of results was tested by Student's paired two-tailed t test.

\section{Results}

All subjects felt that our moving brush provided less sharp but more natural tactility compared with electric stimuli. With either electric or moving tactile stimuli, clear activations in contralateral primary somatosensory cortex (SI) were identified in each subject. However, definite responses in secondary somatosensory cortices were identified in only four and two out of our subjects to electric and tactile stimuli, respectively. In this paper, we presented only the SI responses in various experimental conditions.

Figure 1(b) shows the dipole waveforms of SI responses as a function of time in all subjects. Electric stimuli (intensity 2 ST) on the thumb elicited SEFs with several deflections, peaking on average at $23.8 \pm 0.4 \mathrm{~ms}(\mathrm{~N} 24 \mathrm{mE}$, mean $\pm \mathrm{SEM}), 32.3 \pm 0.9 \mathrm{~ms}$ (P30m), $40.7 \pm 0.9 \mathrm{~ms}(\mathrm{P} 40 \mathrm{~m})$ and $59.2 \pm 2.3 \mathrm{~ms}(\mathrm{P} 55 \mathrm{mE})$. In contrast, SEFs induced by moving tactile stimuli across the thumb consisted of two deflections in six of seven subjects: one early downward and one late upward deflections peaking on average at $24.5 \pm 2.2 \mathrm{~ms}(\mathrm{~N} 24 \mathrm{mT})$ and $53.2 \pm 3.2 \mathrm{~ms}(\mathrm{P} 55 \mathrm{mT})$, respectively.

Figure 1(c) shows the generator of tactile SEFs in Subject 1 located in contralateral SI cortex. This dipole was $27.6 \mathrm{~mm}$ deep 


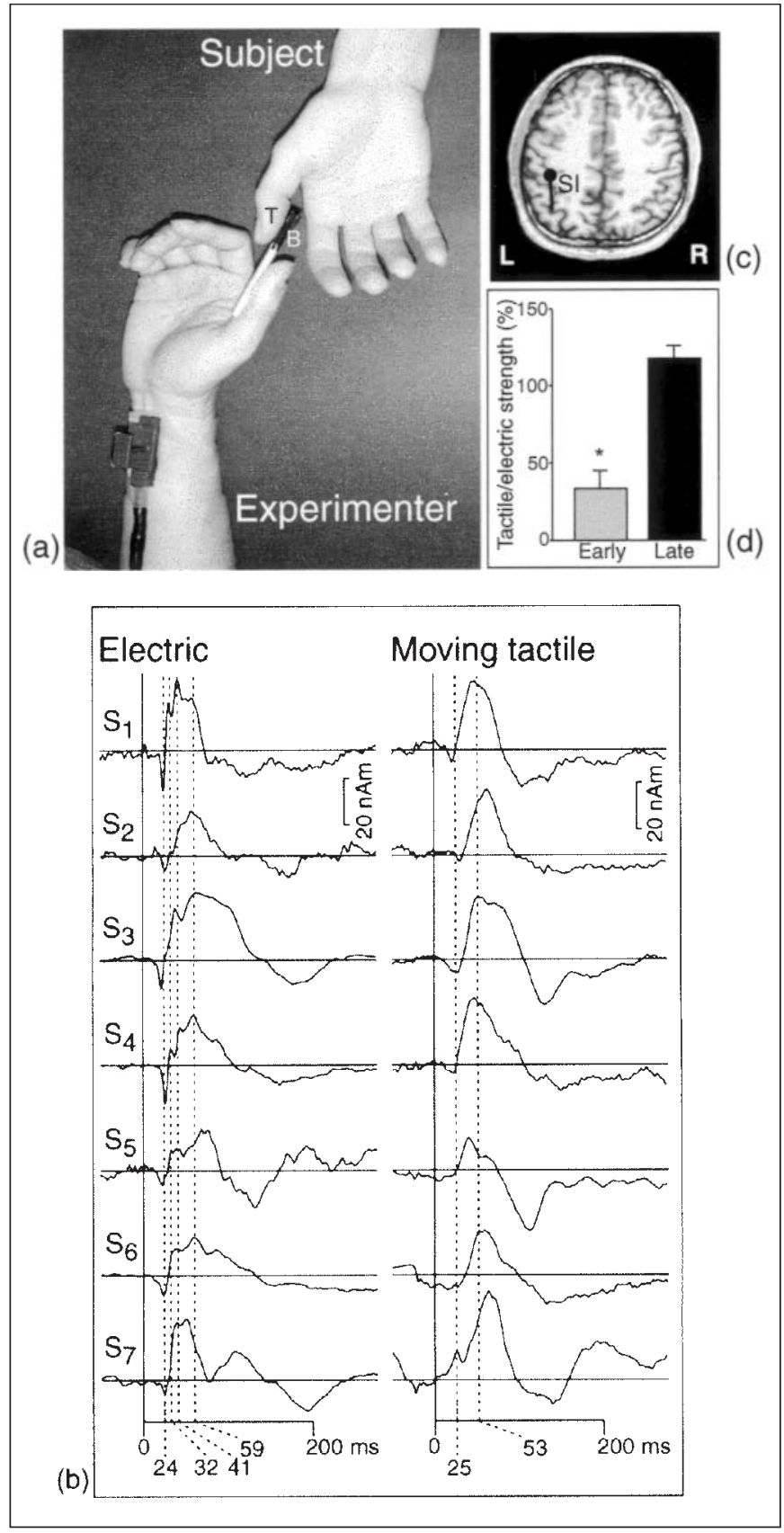

Figure 1(a): Experimental setup for moving tactile stimulation. The palmar surface of the subject's right thumb (upper right) was brushed when the experimenter's right thumb (lower left), with a plastic brush (B) on it, was moving with electric stimulation of the median nerve at the wrist. (b): Source waveforms and strengths in all subjects (S1-S7) as a function of time in contralateral SI cortex to moving tactile and electric stimulation of the right thumb. The dashed lines show the mean latencies at individual main peaks. (c): The source location of SI responses to moving tactile stimuli in Subject 1 superimposed on his own magnetic resonance image. The black line indicates the direction of the source current. $R=$ right, $L=$ left. $(d):$ Mean $(+$ SEM) tactile/electric strength ratio for early ( $N 24 m T$ v.s. $N 24 m E$ ) and late deflections (P55mT v.s. P55mE) of SI responses. Statistical significance $* p<0.01$. below the scalp. The mean depths of SI sources in the seven subjects were $29 \pm 1.7 \mathrm{~mm}$ and $30 \pm 1.2 \mathrm{~mm}$ for electric and tactile SEFs, respectively. For each subject, the generator locations of electric and tactile SEFs were close to each other. The mean coordinate differences between electric and tactile SI responses were $-3.6 \pm 2.1 \mathrm{~mm}$ in $\mathrm{x}$-direction $(\mathrm{p}>0.1),-1.0 \pm 0.8$ $\mathrm{mm}$ in $\mathrm{y}$-direction $(\mathrm{p}>0.1)$, and $2.0 \pm 1.2 \mathrm{~mm}$ in $\mathrm{z}$-direction $(\mathrm{p}$ $>0.1)$. The positive $\mathrm{x}-, \mathrm{y}-$, and $\mathrm{z}$-axes go toward the right preauricular point, the nasion, and the vertex, respectively.

We determined the strengths of the early and late deflections based on the peak amplitude of responses around $24 \mathrm{~ms}$ and 55 ms. Figure 1(d) shows the strength ratios between tactile and
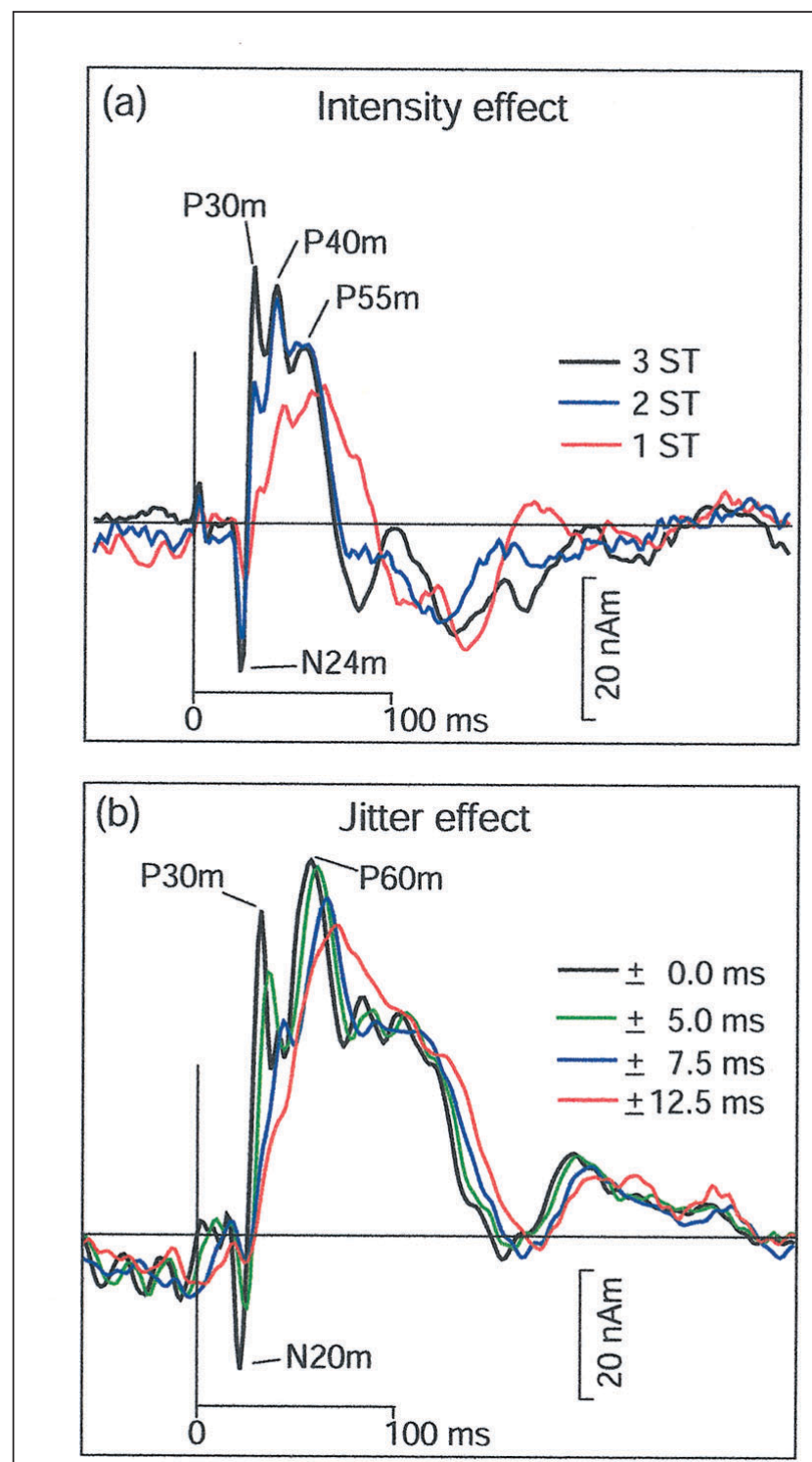

Figure 2(a): Effect of stimulus intensity on the waveforms of SI source as a function of time in Subject 1 to right thumb stimulation. $S T=$ sensory threshold. (b): Effect of time jitter on the waveforms of SI source as a function of time in one subject to electric stimulation ( $9 \mathrm{~mA}$ ) of right median nerve at wrist. 
electric SEFs, separately for the early (N24mT v.s. N24mE) and late (P55mT v.s. P55mE) deflections. The early response was about $65 \%$ smaller to tactile than electric stimuli $(\mathrm{p}<0.01$; against hypothesis of equal amplitude), whereas the late response did not differ between tactile and electric SEFs $(24.0 \pm 2.8 \mathrm{nAm}$ and $20.5 \pm 2.5 \mathrm{nAm}$, respectively; $\mathrm{p}=0.1$ ). The error bars indicate the size of SEM. Moreover, the strength ratios between $\mathrm{N} 24 \mathrm{~m}$ and P55m were $47 \pm 10 \%$ and $15 \pm 0 \%$ for electric and tactile SEFs $(\mathrm{p}<0.01)$, respectively.

Figure 2(a) shows the effect of stimulus intensity on the source waveforms of SI responses to electric stimulation on the right thumb of Subject 1. Compared with the responses at $2 \mathrm{ST}$, the amplitude of $\mathrm{N} 24 \mathrm{mE}$ and $\mathrm{P} 30 \mathrm{mE}$ increased at higher intensity ( $3 \mathrm{ST}$ ), but those of P40mE and P55mE did not change accordingly. With lower intensity (1 ST), the amplitude reduction of $\mathrm{N} 24 \mathrm{mE}, \mathrm{P} 30 \mathrm{mE}$ and $\mathrm{P} 40 \mathrm{mE}(56 \%, 70 \%$ and $50 \%$, respectively) was more prominent than that of the late deflection P55mE (28\%). Moreover, peak latency of N24mE, P30mE and $\mathrm{P} 40 \mathrm{mE}$ decreased clearly as the stimulus intensity increased from $1 \mathrm{ST}$ to $2 \mathrm{ST}$. No further decrease was observed to the stimulus intensity at $3 \mathrm{ST}$.

Figure 2(b) shows the effect of time jitter between individual response epochs on the SI source waveforms to strong electric stimulation $(9 \mathrm{~mA})$ of the right median nerve at the wrist in one author (Y.Y. Lin). The original waveforms (without artificial time jitter) consisted of three clear deflections peaking at $20 \mathrm{~ms}$ (N20mE), $31 \mathrm{~ms}$ (P30mE), and $60 \mathrm{~ms}(\mathrm{P} 60 \mathrm{mE})$. With increased time jitter, the amplitudes of $\mathrm{N} 20 \mathrm{mE}$ and $\mathrm{P} 30 \mathrm{mE}$ dampened progressively, whereas the late $\mathrm{P} 60 \mathrm{mE}$ was relatively preserved. With jitter increasing up to $12.5 \mathrm{~ms}$, P30m disappeared and the overall waveform turned out to be biphasic with $\mathrm{N} 20 \mathrm{~m}$ and P60m, mimicking the waveform of tactile responses.

\section{Discussion}

In line with previous observations, ${ }^{10,14-16}$ we found more than two deflections in the waveforms of electric SI responses. Previous electrophysiological studies in animals have provided evidence that different afferents project to different subregions of SI cortex. ${ }^{17,18}$ Further animal and human studies have shown that electric pulses stimulate various types of peripheral afferent fibers, and thus activate several cytoarchitectonic areas in SI cortex with mixed response waveforms. ${ }^{18-23}$ In contrast, moving tactile SI responses showed simple waveforms with one upward and one downward deflections. The biphasic magnetic responses have been reported in one air-puff study. ${ }^{24}$ Compared with the complex waveforms in electric SEFs, the simpler waveforms in our tactile and previous air-puff studies may be partly related to the relatively selective activation on mechanoreceptors which project largely to area $3 \mathrm{~b}$ of SI cortex. ${ }^{25}$ In the present study, we explored the differences between electric and natural tactile SEFs.

The amplitude of $\mathrm{N} 24 \mathrm{mE}$ was clearly larger than $\mathrm{N} 24 \mathrm{mT}$, but P55mT and P55mE are of about the same size. The different behavior of the early and late responses is in line with previous air-puff studies, ${ }^{9,10}$ suggesting that the early components are more related to types of stimuli and activated receptors. The late components on the other hand are less dependent on physical parameters of sensory stimuli. In our study, effect of electric intensity on the strength of activation was more clearly seen in the early deflection $\mathrm{N} 24 \mathrm{mE}$ than the late deflection $\mathrm{P} 55 \mathrm{mE}$ (Figure 2(a)). Our results are in keeping with previous assumptions that the early SI component reflects the neural coding of stimuli, but the late component may be related to the integrative and cognitive processing of sensory inputs. ${ }^{26,27}$ In addition, we observed the shortening of peak latency of SI responses to higher stimulus intensities ( 2 or $3 \mathrm{ST}$ ), in line with one previous report. ${ }^{28}$

The smaller amplitude ratio of the $\mathrm{N} 24 \mathrm{~m} / \mathrm{P} 55 \mathrm{~m}$ deflections for tactile than electric SEFs agreed with one previous study showing that spatial parameters of sensory stimuli are most intimately related to the amplitude of the early (N20-P27) components. ${ }^{9}$ The same size of responses can be generated by activation of a rather small group of cutaneous fibers. This idea was also supported by the clear dampening of $\mathrm{N} 24 \mathrm{mE}$ and P30mE but preservation of the late deflection at lower stimulus intensity (Figure 2(a)). It thus seems feasible that of all the afferent fibers conveying the early deflection, the lower threshold components participate the generation of the late deflection. ${ }^{29,30}$ Also, the lesser sensitivity of the late component to the changes in stimulus intensity may be partly because it is more related to the integrative and cognitive processing rather than the intensity coding of sensory input. ${ }^{26,27}$

Although we carefully tried to keep the contact of the brush with the subject's thumb stable during the whole measurement, we could not rule out the size variation of stimulated skin area or the time variation of effective stimulus strength between epochs. One possible explanation for the small size of the tactile $\mathrm{N} 24 \mathrm{~m}$ would be increased jitter between stimulus onset. In fact, our simulation experiment showed that the effect of time jitter was more clearly seen on the early deflection than the late deflection (Figure 2(b)). Thus moving tactile stimuli across thumb skin may exert temporal and spatial integration of the somatosensory responses ${ }^{31}$ with consequent changes of waveforms.

To our knowledge, no mechanically operated moving brush has been used for studying neuromagnetic cortical responses. In the present study, the experimenter's thumb movements elicited by electric stimulation of the experimenter's median nerve provided a near natural tactile stimuli to the subjects. In the future, a mechanically operated design may be expected to provide moving stimuli with less jitter and more consistent stimulus intensity.

\section{Conclusion}

The electric and moving tactile stimulation of the right thumb elicited SEFs in all subjects, and the activated SI sources were close to each other. The waveforms of tactile responses were simple with a small early deflection (N24mT) and a clear late deflection (P55mT). Decreasing intensity or increasing jitter between epochs changed electric SEF waveforms so that they resembled tactile SEFs with a smaller proportion of the early deflection. We propose that of all the afferent fibers conveying the early deflection, the lower threshold components participate in the generation of the late deflection.

\section{ACKNOWLEDGEMENTS}

This work was supported in part by the Academy of Finland, and research grants VGH-90-443 and VGH-90-370 from Taipei Veterans General Hospital (Y.Y.L.), Taipei, Taiwan. We thank Professor Riitta 
Hari for valuable comments on the manuscript. We also thank Dr. Peter Berglund, Mr. Ronny Schreiber and Dr. Sari Avikainen for technical assistance.

\section{REFERENCES}

1. Hari R, Forss N. Magnetoencephalography in the study of human somatosensory cortical processing. Philos Trans R Soc Lond B Biol Sci 1999; 354: 1145-1154.

2. Franzén O, Offenloch K. Evoked response correlates of psychophysical magnitude estimates for tactile stimulation in man. Exp Brain Res 1969; 8: 1-18.

3. Nakanishi T, Takita K, Toyokura Y. Somatosensory evoked responses to tactile tap in man. Electroencephalogr Clin Neurophysiol 1973; 34: 1-6.

4. Jousmäki V, Hari R. Somatosensory evoked fields to large-area vibrotactile stimuli. Clin Neurophysiol 1999; 110: 905-909.

5. Sears TA. Action potentials evoked in digital nerves by stimulation of mechanoreceptors in the human finger. J Physiol (Lond.) 1959; 148: $30-31$.

6. Matsumiya Y, Mostofsky DI. Somatosensory evoked responses elicited by corneal and nostril air puff stimulation. Electroencephalogr Clin Neurophysiol 1972; 33: 225-227.

7. Gardner EP, Hämäläinen HA, Warren S, Davis J, Young W. Somatosensory evoked potentials (SEPs) and cortical single unit responses elicited by mechanical tactile stimuli in awake monkeys. Electroencephalogr Clin Neurophysiol 1984; 58: 537552.

8. Schieppati M, Ducati A. Short latency cortical potentials evoked by tactile air-jet stimulation of body and face in man. Electroencephalogr Clin Neurophysiol 1984; 58: 418-425.

9. Hashimoto I. Somatosensory evoked potentials elicited by air-puff stimuli generated by a new high-speed air control system. Electroencephalogr Clin Neurophysiol 1987; 67: 231-237.

10. Forss N, Salmelin R, Hari R. Comparison of somatosensory evoked fields to airpuff and electric stimuli. Electroencephalogr Clin Neurophysiol 1994; 92: 510-517.

11. Hari R, Jousmäki V, Vanni S. Kohti inhimillisempää aivotutkimusta: PAH. Duodecim 1996; 112: 2210-2214.

12. Lin YY, Simoes C, Forss N, Hari R. Differential effects of muscle contraction from various body parts on neuromagnetic somatosensory responses. Neuroimage 2000; 11: 334-340.

13. Hämäläinen M, Hari R, Ilmoniemi RJ, Knuutila J, Lounasmaa OV. Magnetoencephalography - theory, instrumentation, and applications to noninvasive studies of the working human brain. Rev Mod Phy 1993; 65: 413-497.

14. Hari R, Reinikainen K, Kaukoranta E, et al. Somatosensory evoked cerebral magnetic fields from SI and SII in man. Electroencephalogr Clin Neurophysiol 1984; 57: 254-263.

15. Okada Y, Tanenbaum R, Williamson S, Kaufman L. Somatotopic organization of the human somatosensory cortex as revealed by magnetic measurements. Exp Brain Res 1984; 56: 197-205.

16. Wood CC, Cohen D, Cuffin BN, Yarita M, Allison T. Electric sources in the human somatosensory cortex: identification by combined magnetic and potential field recordings. Science 1985; 227: 1051-1053.

17. Powell TPS, Mountcastle VB. Some aspects of the functional organization of the postcentral gyrus of the monkey: a correlation of findings obtained in a single unit analysis with cytoarchitecture. Bull Johns Hopkins Hosp 1959; 105: 133-162.

18. Kaas JH, Nelson RJ, Sur M, Lin CS, Merzenich MM. Multiple representations of the body within the primary somatosensory cortex of primates. Science 1979; 204: 521-523.

19. Phillips CG, Powell TP, Wiesendanger M. Projection from lowthreshold muscle afferents of hand and forearm to area $3 \mathrm{a}$ of baboon's cortex. J Physiol 1971; 217: 419-446.

20. Jones SJ, Power CN. Scalp topography of human somatosensory evoked potentials: the effect of interfering tactile stimulation applied to the hand. Electroencephalogr Clin Neurophysiol 1984; 58: 25-36.

21. Pons TP, Garraghty PE, Cusick CG, Kaas JH. The somatotopic organization of area 2 in macaque monkeys. J Comp Neurol 1985; 241: 445-466.

22. Kaukoranta E, Hamalainen M, Sarvas J, Hari R. Mixed and sensory nerve stimulations activate different cytoarchitectonic areas in the human primary somatosensory cortex SI. Neuromagnetic recordings and statistical considerations. Exp Brain Res 1986; 63: 60-66.

23. Allison T, McCarthy G, Wood CC, et al. Human cortical potentials evoked by stimulation of the median nerve. I. Cytoarchitectonic areas generating short-latency activity. J Neurophysiol 1989; 62: 694-710

24. Huttunen J. Magnetic cortical responses evoked by tactile stimulation of the middle finger in man. Pflügers Arch 1986; 407: 129-133.

25. Kaas JH. The organization of somatosensory cortex in primates and other mammals. In: Euler $\mathrm{C}$ von, Franzen $\mathrm{O}$, Lindblom $\mathrm{O}$, Ottoson D, (Eds). Somatosensory Mechanisms. London: Macmillan Press; 1984: 51-59.

26. Desmedt JE, Huy NT, Bourguet M. The cognitive P40, N60 and P100 components of somatosensory evoked potentials and the earliest electrical signs of sensory processing in man. Electroencephalogr Clin Neurophysiol 1983; 56: 272-282.

27. Hashimoto I, Yoshikawa K, Sasaki M. Exp Brain Res 1988; 73: 459469.

28. Jousmaki V, Forss N. Effects of stimulus intensity on signals from human somatosensory cortices. Neuroreport 1998; 9: 3427-3431.

29. Tsuji S, Shibasaki H, Kato M, Kuroiwa Y, Shima F. Subcortical, thalamic and cortical somatosensory evoked potentials to median nerve stimulation. Electroencephalogr Clin Neurophysiol 1984; 59: 465-476.

30. Huttunen J. Effects of stimulus intensity on frontal, central and parietal somatosensory evoked potentials after median nerve stimulation. Electromyogr Clin Neurophysiol 1995; 35: 217-223.

31. Gardner EP, Costanzo RM. Temporal integration of multiple-point stimuli in primary somatosensory cortical receptive fields of alert monkeys. J Neurophysiol 1980; 43: 444-468. 